Pacific Journal of Mathematics

GENERA IN NORMAL EXTENSIONS 


\title{
GENERA IN NORMAL EXTENSIONS
}

\author{
ROBERT GOLD
}

Let $K / F$ be a finite normal extension of algebraic number fields and let $C_{K}$ be the ideal class group of $K$. There are two fundamentally different ways to define the principal genus of $C_{E}$ with respect to $F$. Classically the principal genus is described by norm residue symbols. By the modern definition it is the class group of the maximal unramified extension of $K$ which is the composite of $K$ with an abelian extension of $F$. It is shown here that the two definitions are equivalent.

Let $F$ be a finite algebraic number field and $K$ a finite normal extension of $F$ with $G=\mathrm{Gal}(K / F)$. Let $\bar{K}$ be the Hilbert class field of $K$ and let $C_{K}$ be the ideal class group of $K$. By class field theory the fields lying between $K$ and $\bar{K}$ are in one-one correspondence with the subgroups of $C_{K}$. (See [3] or [4] for the class field theory involved.) Let $L$ be the genus field for $K / F$. As defined by Fröhlich ([1]), $L$ is the composite of $K$ with the maximal abelian extension of $F$ in $\bar{K}$. Calling this maximal abelian extension $E$, we have $\bar{K} \supseteqq L=K E \supseteqq K, E \supseteqq F$ and $K \cap E$ is the maximal abelian extension of $F$ in $K$. The subgroup of $C_{K}$ corresponding to $L$ is the principal genus of $C_{K}$. Gauss's definition of the principal genus is based on arithmetic characters. In [2] we showed that when $G$ is abelian the two definitions are equivalent. Here we will show that in fact they are equivalent for any $G$.

Let $C_{F}$ be the ideal class group of $F$ and let $N_{K / F}: C_{K} \rightarrow C_{F}$ be the norm map on ideal class groups. Let $\bar{F}$ be the Hilbert class field of $F$ and ${ }_{N} C_{K}$ the kernel of the norm map. Then the subgroup ${ }_{N} C_{K}$ of $C_{K}$ corresponds to the extension $K \bar{F}$ of $K$. Clearly $L \supseteqq K \bar{F}$ and, letting $H$ denote the principal genus of $C_{K}$, we see that ${ }_{N} C_{K} \supseteq H$.

We now proceed to describe the characters in Gauss's definition. Let $P_{1}, \cdots, P_{t}$ be the primes of $K$, finite or infinite, ramified in $K / F$. For each $i$ choose a prime $\bar{P}_{i}$ in $\bar{K}$ such that $\bar{P}_{i} \cap K=P_{i}$. This allows a consistent choice of primes in each subfield $k$ by $P_{k, i}=$ $\bar{P}_{i} \cap k$. And we will denote the completed localization of $k$ at $P_{k, i}$ by $k_{i}$. In particular we have the chain $\bar{K}_{i} \supseteqq L_{i} \supseteqq K_{i}, E_{i} \supseteqq F_{i}$ of local fields. For an ideal $\mathfrak{A}$ of a field $k$ let [भ] denote the ideal class of $\mathfrak{A}$. Now let $\mathfrak{A}$ be an ideal of $K$ such that $[\mathfrak{U}] \in_{N} C_{K}$. Thus $N_{K / F}(\mathfrak{U})$ is a principal ideal of $F$, say $N_{K / F}(\mathfrak{O})=(a), a \in F$. For each $i$ we have a norm residue symbol $\left(\left(K_{i} / F_{i}\right) / a\right)$ which we will also write $\left((a, K / F) / P_{i}\right)$ or most simply $\chi_{i}(a)$. This symbol is an element 
of the local group $\operatorname{Gal}\left(K_{i} / F_{i}\right)$ modulo its commutator. We may identify $\operatorname{Gal}\left(K_{i} / F_{i}\right)$ with the decomposition group $Z_{i}$ of $P_{i}$ in $K / F$. Thus we have a homomorphism $\chi_{i}: F^{\times} \rightarrow Z_{i} /\left[Z_{i}, Z_{i}\right]=Z_{i}^{a b}$. Let $\chi_{:} F^{\times} \rightarrow$ $\prod_{i=1}^{t} Z_{i}^{a b}$ by $\chi(a)=\left(\chi_{1}(a), \cdots, \chi_{t}(a)\right)$. Let $U_{F}$ denote the units of $F, P_{F}$ the principal ideals of $F$, and $S=\chi\left(U_{F}\right)$. Then $\chi$ induces a homomorphism which we'll also denote by $\chi_{:} P_{F}=F^{\times} / U_{F} \rightarrow \prod_{i=1}^{t} Z_{t}^{a b} / S$. Let $(a) \in P_{F}$ and $(a)=\left(N_{K / F}(b)\right)=N_{K / F}((b))$. Then $a=\varepsilon \cdot N_{K / F}(b)$ for some $\varepsilon \in U_{F}$ and $\chi_{i}(a)=\chi_{i}(\varepsilon) \chi_{i}\left(N_{K / F}(b)\right)=\chi_{i}(\varepsilon)$ for $i=1, \cdots, t$ since $\chi_{i}\left(N_{K / F}(b)\right)=\left(\left(K_{i} / F_{i}\right) / N_{K / F}(b)\right)$ and every global norm is a local norm everywhere. It follows that $\chi: P_{F} \rightarrow \prod Z_{i}^{a b} / S$ vanishes on $N_{K / F}\left(P_{K}\right) \subseteq P_{F}$. Note that $N_{K / F}:{ }_{N} C_{K} \rightarrow P_{F} / N\left(P_{K}\right)$ since if $[\mathfrak{U}]=[\mathfrak{B}] \in{ }_{N} C_{K}$ then $\mathfrak{U}=(\alpha) \mathfrak{B}$ and $N(\mathfrak{U})=N((\alpha)) N(\mathfrak{B}) \in P_{F}$. Now we can define $f=\chi_{\circ} N_{K / F}:{ }_{N} C_{K} \rightarrow$ $P_{F} / N\left(P_{K}\right) \rightarrow \amalg Z_{i}^{a b} / S$. The formal statement of the equivalence of the two definitions of principal genus is given by

THEOREM. Let $K / F$ be a finite normal extension of number fields and let $H$ be the principal genus in the sense of Fröhlich. Let $f:{ }_{N} C_{K} \rightarrow \prod_{i=1}^{t} Z_{i}^{a b} / S$ be the modified product of local norm residue symbols described above. Then $H=\operatorname{Ker}(f)$.

Proof. First we show that $\operatorname{Ker}(f) \cong H$. Let $P$ be a prime ideal of $K, P \neq P_{i}, i=1, \cdots, t ;[P] \in \operatorname{Ker}(f)$, and $P$ of absolute degree 1 . Since $[P] \in \operatorname{Ker}(f)$ and $P$ is of degree $1, N_{K / F}(P)=\mathfrak{p}=(\rho)$ where $\mathfrak{p}=P \cap F$ and $\rho \in F$. Moreover $\rho$ may be chosen so that $\chi_{i}(\rho)=1$, $i=1, \cdots t$, since $\rho$ times any unit of $F$ generates $\mathfrak{p}$ and $[P] \in \operatorname{Ker}(f)$ implies $\chi(\rho)=\chi(\varepsilon)$ for some $\varepsilon \in U_{F}$.

Let $M_{i}$ be the maximal abelian extension of $F_{i}$ in $L_{i}$. So $K_{i} \cap M_{i}$ is the maximal abelian extension of $F_{i}$ in $K_{i}$. Then

$$
\left.\left(\frac{M_{i} \mid F_{i}}{\rho}\right)\right|_{M_{i} \cap K_{i}}=\left(\frac{M_{i} \cap K_{i} \mid F_{i}}{\rho}\right)=\left(\frac{K_{i} \mid F_{i}}{\rho}\right)=\chi_{i}(\rho)=1 .
$$

The second equality here follows from the fact that $N_{K_{i^{\prime}} F_{i}}\left(K_{i}\right)=$ $N_{K_{i} \cap M_{i} / F_{i}}\left(K_{i} \cap M_{i}\right)$. Therefore

$$
\left(\frac{M_{i} / F_{i}}{\rho}\right) \in \operatorname{Gal}\left(M_{i} / M_{i} \cap K_{i}\right) \subseteq \operatorname{Gal}\left(M_{i} / F_{i}\right) .
$$

Since $P \neq P_{i}$, any $i, \rho$ is a $P_{i}$-unit for each $i$. Thus $\left(\left(M_{i} / F_{i}\right) / \rho\right) \in$ $T\left(M_{i} / F_{i}\right) \subseteq \operatorname{Gal}\left(M_{i} / F_{i}\right)$ where $T$ is the inertia group of the local extension. So we have

$$
\left(\frac{M / F_{i} F_{i}}{\rho}\right) \in T\left(M_{i} / F_{\imath}\right) \cap \operatorname{Gal}\left(M_{i} / M_{i} \cap K_{i}\right)=T\left(M /{ }_{i} M_{i} \cap K_{i}\right)
$$

Lemma. $L_{i} / F_{i}$ be a normal extension of local fields and $M_{i}$ the 
maximal abelian extension of $F_{i}$ in $L_{i}$. If $L_{i} \supseteqq K_{i} \supseteqq F_{i}$ and $L_{i} / K_{i}$ is unramified, then $M_{i} / M_{i} \cap K_{i}$ is unramified.

The lemma, to be proved below, implies that $T\left(M_{i} / M_{i} \cap K_{i}\right)=\{1\}$ and therefore $\left(\left(M_{i} / F_{i}\right) / \rho\right)=1$. Since $M_{i} \supseteqq E_{i}$ it follows that $\left(\left(E_{i} / F_{i}\right) / \rho\right)=$ $1=\left((\rho, E / F) / P_{i}\right)$ for all $i$. So we have $E / F$ abelian, $\rho \in F$, and $\left((\rho, E / F) / \mathfrak{p}_{i}\right)=1$ for $\mathfrak{p}_{i}=F \cap P_{i}, i=1, \cdots, t$. Since $\bar{K} \supseteqq E$, the $\left\{\mathfrak{p}_{i}\right\}$ includes all primes of $F$ ramified in $E / F$. For every unramified prime of $F$ at which $\rho$ is a unit the norm residue symbol is 1 . The only undetermined symbol is $((\rho, E / F) / \mathfrak{p})$. By the product formula for norm residue symbols, the product of all symbols is 1 . Hence we must have $((\rho, E / F) / \mathfrak{p})=1$. Recall that $(\rho)=\mathfrak{p}$, i.e. $\rho$ is a prime element at $\mathfrak{p}$, and $\mathfrak{p}$ is unramified in $E / F$. Hence $((\rho, E / F) / \mathfrak{p})$ generates the decomposition group of $\mathfrak{p}$ in $E / F$. We conclude that $\mathfrak{p}$ is completely decomposed in $E / F$. It follows by standard arguments that $P$ is completely decomposed in $L / K$ since $L=K E$. The subgroup of $C_{K}$ corresponding to a subfield $k$ of $\bar{K}$ can be characterized as the classes of all prime ideals of $K$ which are completely decomposed in $k / K$. Thus $[P] \in H$ since $H$ corresponds to $L$.

Now we show that $\operatorname{Ker}(f) \supseteqq H$. Let $P$ be a prime of $K$ of absolute degree 1 with $[P] \in H$. Let $N_{K / F}(P)=\mathfrak{p}=(\rho), \rho \in F$ and as above let $P_{\imath}, i=1, \cdots, t$ be the primes of $K$ ramified in $K / F$. We may assume also $P \neq P_{i}$ for any $i$. Since $[P] \in H, P$ is completely decomposed in $L / K$. Say, $P=Q_{1} \cdots Q_{g}$ so that $N_{L / F}\left(Q_{1}\right)=(\rho)$. Let $\mathfrak{m}$ be a divisor of $F$ divisible by high powers of all $P_{i}$ and prime to $P$. Since $E$ is the maximal abelian extension of $F$ in $L$ and in $\bar{K}$ the norm limitation theorem implies that

$$
\text { (*) } \quad N_{E / F}\left(I_{\mathrm{m}}(E)\right) \cdot S_{\mathrm{m}}(F)=N_{L / F}\left(I_{\mathrm{m}}(L)\right) \cdot S_{\mathrm{m}}(F)=N_{\bar{K} / F}\left(I_{\mathrm{m}}(\bar{K})\right) \cdot S_{\mathrm{m}}(F)
$$

where $I_{\mathrm{m}}(k)$ is the group of ideals of $k$ relatively prime to $\mathfrak{m}$ and $S_{\mathrm{m}}(k)$ is the ideal ray (Strahl) $\bmod \mathfrak{m}$.

We have noted that $(\rho)=N_{L / F}(Q)$ with $Q_{i} \in I_{\mathrm{m}}(L)$. It follows from $(*)$ that we can write $(\rho)=N_{\bar{K} / F}(\mathfrak{N}) \cdot(\alpha)$ where $\mathfrak{A} \in I_{\mathrm{m}}(\bar{K})$ and $(\alpha) \in S_{\mathrm{m}}(F)$. The norm from $\bar{K}$ to $K$ of any ideal of $\bar{K}$ is a principal ideal of $K$. Let $N_{\bar{K} / K}(\mathfrak{U})=(a), a \in K$. So $(\rho)=(\alpha) N_{\bar{K} / F}(\mathfrak{Q})=$ $(\alpha) N_{K / K}\left(N_{E / K}(\mathfrak{X})\right)=(\alpha)\left(N_{K / F}(a)\right)$ or $\varepsilon \rho=\alpha \cdot N_{K / F}(a)$ for some unit $\varepsilon \in U_{F}$. Therefore

$$
\left(\frac{\varepsilon \rho, K / F}{P_{i}}\right)=\left(\frac{\alpha, K / F}{P_{i}}\right) \cdot\left(\frac{N_{K / F}(a), K / F}{P_{i}}\right) .
$$

Since a global norm is certainly a local norm $\left(\left(N_{K / F}(a), K / F\right) / P_{i}\right)=1$. Also since $\alpha \in F, \alpha \equiv 1(\mathfrak{m})$ and $\mathfrak{m}$ is divisible by high powers of the 
$P_{i}$ we see that $\left(\left(M_{i} \cap K_{i} / F_{i}\right) / \alpha\right)=1$. And therefore

$$
\left(\frac{K_{i} / F_{i}}{\alpha}\right)=\left(\frac{\alpha, K / F}{P_{i}}\right)=1 \text {. }
$$

Thus $\left((\varepsilon \rho, K / F) / P_{i}\right)=1$ for all $i$. In other words $\chi(\rho)=\chi\left(\varepsilon^{-1}\right)$, which gives $[P] \in \operatorname{Ker}(f)$.

Proof of the lemma. Let $T(L / F)$ be the inertia subgroup of $\operatorname{Gal}(L / F)$. The quotient $\operatorname{Gal}(L / F) / T(L / F)$ is a cyclic group, hence $T(L / F)$ contains the commutator subgroup of $\mathrm{Gal}(L / F)$, which is Gal $(L / M)$. Thus $L / M$ is totally ramified. Letting $e$ denote the ramification index, we have $e(L / K \cap M) \geqq[L: M] \geqq[K: K \cap M]$. This last inequality follows from the fact that $L \supseteqq K M$ and, since $M / K \cap M$ is galois, $[K M: M]=[K: K \cap M]$. Since $L / K$ is unramified, $e(L / K \cap M) \leqq$ $[K: K \cap M]$. Therefore $e(L / K \cap M)=[K: K \cap M]=[L: M]=e(L / M)$ and so $e(M / K \cap M)=1$.

\section{REFERENCES}

1. A. Fröhlich, The genus field and genus group in finite number fields, I, II, Mathematika, 6 (1959), 40-46, 142-146.

2. R. Gold, Genera in abelian extensions, Proc. Amer. Math. Soc., 47 (1975), 25-28.

3. H. Hasse, Vorlesungen über Klassenkörpertheorie, Physica-Verlag, Würzburg, 1967.

4. G. Janusz, Algebraic Number Fields, Academic Press, New York, 1973.

Received July 17, 1975.

Ohio State University 


\title{
PACIFIC JOURNAL OF MATHEMATICS
}

\author{
EDITORS
}

\author{
RICHARD ARENS (Managing Editor) \\ University of California \\ Los Angeles, California 90024
}

\author{
R. A. Beaumont \\ University of Washington \\ Seattle, Washington 98105
}

J. DugundjI

Department of Mathematics

University of Southern California

Los Angeles, California 90007

D. Gilbarg and J. Milgram

Stanford University

Stanford, California 94305

\section{ASSOCIATE EDITORS}
E. F. BECKENBACH
B. H. NEUMANN
F. WOLF
K. Yoshida

\section{SUPPORTING INSTITUTIONS}

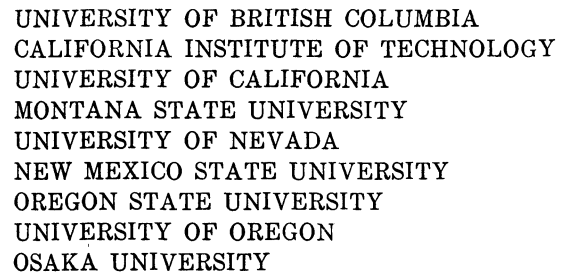

UNIVERSITY OF BRITISH COLUMBIA CALIFORNIA INSTITUTE OF TECHNOLOGY UNIVERSITY OF CALIFORNIA MONTANA STATE UNIVERSITY UNIVERSITY OF NEVADA NEW MEXICO STATE UNIVERSITY OREGON STATE UNIVERSITY UNIVERSITY OF OREGON OSAKA UNIVERSITY

\author{
UNIVERSITY OF SOUTHERN CALIFORNIA \\ STANFORD UNIVERSITY \\ UNIVERSITY OF HAWAII \\ UNIVERSITY OF TOKYO \\ UNIVERSITY OF UTAH \\ WASHINGTON STATE UNIVERSITY \\ UNIVERSITY OF WASHINGTON

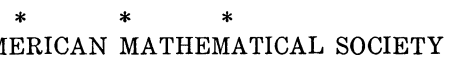

The Supporting Institutions listed above contribute to the cost of publication of this Journal, but they are not owners or publishers and have no responsibility for its content or policies.

Mathematical papers intended for publication in the Pacific Journal of Mathematics should be in typed form or offset-reproduced, (not dittoed), double spaced with large margins. Please do not use built up fractions in the text of your manuscript. You may however, use them in the displayed equations. Underline Greek letters in red, German in green, and script in blue. The first paragraph or two must be capable of being used separately as a synopsis of the entire paper. Items of the bibliography should not be cited there unless absolutely necessary, in which case they must be identified by author and Journal, rather than by item number. Manuscripts, in triplicate, may be sent to any one of the editors. Please classify according to the scheme of Math. Reviews, Index to Vol. 39. All other communications should be addressed to the managing editor, or Elaine Barth, University of California, Los Angeles, California, 90024.

The Pacific Journal of Mathematics expects the author's institution to pay page charges, and reserves the right to delay publication for nonpayment of charges in case of financial emergency.

100 reprints are provided free for each article, only if page charges have been substantially paid. Additional copies may be obtained at cost in multiples of 50 .

The Pacific Journal of Mathematics is issued monthly as of January 1966. Regular subscription rate: $\$ 72.00$ a year (6 Vols., 12 issues). Special rate: $\$ 36.00$ a year to individual members of supporting institutions.

Subscriptions, orders for back numbers, and changes of address should be sent to Pacific Journal of Mathematics, 103 Highland Boulevard, Berkeley, California, 94708.

PUBLISHED BY PACIFIC JOURNAL OF MATHEMATICS, A NON-PROFIT CORPORATION

Printed at Kokusai Bunken Insatsusha (International Academic Printing Co., Ltd.), 8-8, 3-chome, Takadanobaba, Shinjuku-ku, Tokyo 160, Japan.

Copyright (C) 1975 by Pacific Journal of Mathematics Manufactured and first issued in Japan 


\section{Pacific Journal of Mathematics}

\section{Vol. 63, No. 2 \\ April, 1976}

Joseph Anthony Ball and Arthur R. Lubin, On a class of contractive perturbations

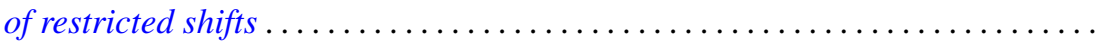

Joseph Becker and William C. Brown, On extending higher derivations generated

by cup products to the integral closure .......................

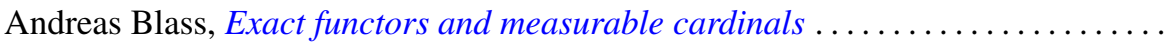

Joseph Eugene Collison, A variance property for arithmetic functions . . . . . . . . . .

Craig McCormack Cordes, Quadratic forms over nonformally real fields with a

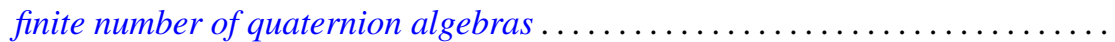

Freddy Delbaen, Weakly compact sets in $H^{1} \ldots \ldots \ldots \ldots \ldots \ldots \ldots \ldots \ldots$

G. D. Dikshit, Absolute Nörlund summability factors for Fourier series ..........

Edward Richard Fadell, Nielsen numbers as a homotopy type invariant. . ........

Josip Globevnik, Analytic extensions of vector-valued functions . . . . . . . . . . . .

Robert Gold, Genera in normal extensions . . . . . . . . . . . . . . . . . . . 389

Solomon Wolf Golomb, Formulas for the next prime

Robert L. Griess, Jr., The splitting of extensions of $S L(3,3)$ by the vector space

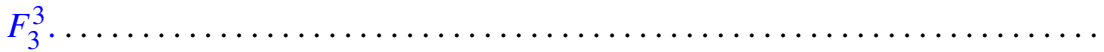

Thomas Alan Keagy, Matrix transformations and absolute summability .........

Kazuo Kishi, Analytic maps of the open unit disk onto a Gleason part.

Kwangil Koh, Jiang Luh and Mohan S. Putcha, On the associativity and commutativity of algebras over commutative rings . ..... . .

James C. Lillo, Asymptotic behavior of solutions of retarded differential difference equations.

John Alan MacBain, Local and global bifurcation from normal eigenvalues ..

Anna Maria Mantero, Sets of uniqueness and multiplicity for $L^{p}$

J. F. McClendon, Embedding metric families

L. Robbiano and Giuseppe Valla, Primary powers of a prime ideal .

Wolfgang Ruess, Generalized inductive limit topologies and barrelledness

properties.

Judith D. Sally, Bounds for numbers of generators of Cohen-Macaulay ideals

Helga Schirmer, Mappings of polyhedra with prescribed fixed points and fixed point indices.

Cho Wei Sit, Quotients of complete multipartite graphs

S. Sznajder and Zbigniew Zielezny, Solvability of convolution equations in $\mathscr{K}_{p}^{\prime}$,

$p>1$.

Mitchell Herbert Taibleson, The existence of natural field structures for finite

dimensional vector spaces over local fields

William Yslas Vélez, A characterization of completely regular fields

P. S. Venkatesan, On right unipotent semigroups ..............

Kenneth S. Williams, A rational octic reciprocity law ............

Robert Ross Wilson, Lattice orderings on the real field .......... 